\title{
Uso de nuevas tecnologías y rendimiento académico en una muestra de bachilleres de 16 a 18 años. Un estudio exploratorio Use of new technologies and academic performance in a sample of 16-18 years old scholars. An exploratory study
}

\author{
Ana Mónica Chérrez Bermejo \\ monicacherrez1970@gmail.com \\ Departamento de Metodología de las \\ Ciencias del Comportamiento \\ Facultad de Psicología, UNED, España \\ Pamplona, España
}

\begin{abstract}
Resumen- Las nuevas tecnologías acentúan una brecha generacional y cultural mientras ofrece nuevas posibilidades y genera riesgos para los jóvenes actuales. Es frecuente considerar el uso de TIC como un factor de disminución del rendimiento escolar. Objetivo: Esta investigación mide las posibles diferencias de género en el uso y frecuencia de las nuevas Tecnologías de la Información y la Comunicación en estudiantes de bachillerato. Explora también su posible asociación con una disminución del rendimiento escolar.Material y métodos: Muestra aleatoria secuencial de 72 estudiantes españoles de bachillerato de ambos sexos, con edades comprendidas entre 16 y 18 años, usuarios de TIC en sus hábitos diarios de ocio. Los test estadísticos empleados fueron la " $\mathrm{t}$ " de Student y ANOVA bifactorial.Resultados: Mayor uso de internet y móvil en chicas, y de videojuegos en chicos. Relación débil y negativa entre rendimiento y adicción. Conclusión: Se constatan diferencias de género en el tipo de dispositivo usado. No se hallaron diferencias significativas de género en el desarrollo de un cuadro de adicción a TIC. No se atribuye disminución del rendimiento en ningún individuo de la muestra escolar por conexiones diarias por ocio a las TIC.
\end{abstract}

\section{Palabras clave: Adicción, uso, tecnología.}

Abstract- New technologies accentuate a generational and cultural gap while offering new possibilities and creating risks for today's youth. It is common to consider the use of ICT as a factor in reducing school performance. Objective: This research measures the possible gender differences in the use and frequency of the new Information and Communication Technologies in high school students. It also explores its possible association with a decrease in school performance. Material and methods: Sequential random sample of 72 Spanish high school students of both sexes, aged between 16 and 18 years, ICT users in their daily leisure habits. The statistical tests used were Student's "t" and bifactorial ANOVA. Results: Greater use of internet and mobile phones in girls, and video games in boys. Weak and negative relationship between performance and addiction. Conclusion: Gender differences are found in the type of device used. No significant gender differences were found in the development of an ICT addiction picture. No performance decrease is attributed to any individual in the school sample due to daily connections for leisure to ICT.

Keywords: Addiction, use, technology.

\section{INTRODUCCIÓN}

El uso de las Tecnologías de la Información y la Comunicación (TIC) forma parte de los hábitos cotidianos de niños, adolescentes y jóvenes. Lejos queda ya la denominada Sociedad de la Información que arrancaba con los cambios tecnológicos de los años 70 en el siglo XX. La presencia de las TIC en múltiples esferas de la vida diaria constituye un fenómeno irreversible en todas las edades. Incluso algunos expertos defienden actualmente que el proceso de digitalización está cambiando en cierta medida el comportamiento de la especie humana.

Esta transformación afecta a nuestra forma de aprender, y abarca todos los ámbitos de nuestra cultura (Castañeda et al., 2013; Tourón et al., 2013). Uno de los condicionantes de este cambio cultural es la denominada «brecha digital generacional» (Prensky 2010; Robles et al., 2016; TorresAlbero et al., 2017). Según ponen de relieve algunas investigaciones recientes, las habilidades digitales de los ciudadanos están relacionadas con el nivel de estudios, la renta, y también con el género (Robles y Torres-Albero, 2016).

A continuación, vamos a centrarnos en el análisis de diversos trabajos publicados que analizan la relación existente entre un uso excesivo de las TIC y su repercusión en el rendimiento escolar. Diversas investigaciones internacionales han explorado en el ámbito de los beneficios y riesgos potenciales que conllevan las TIC (Ballesteros y Megías, 2015; Bringué y Sádaba, 2009; Martínez y González, 2018). Tal y como señala el estudio de Ballesteros y Megías (2015) los jóvenes que dedican su tiempo a las redes sociales afirman que dedica menos tiempo a leer (44\%) y a ver la Tv (44\%) y, algo menos, a dormir (31\%). Reconocen también que las TIC afectan a campos relevantes para el aprendizaje o la salud, y encarnan buena parte de las preocupaciones de las personas adultas en relación a sus hijos e hijas. En el caso de la Generación Interactiva en Iberoamérica, Bringué y Sábada (2009) constatan que, en el grupo de edad comprendido entre los 10 y 18 años, el $23 \%$ de menores navega por internet sin mediación del adulto. Estos porcentajes aumentan con la edad y se sitúan en el $48 \%$ en el caso de las chicas por encima de 18 
años. Además, el 25\% declara ponerse nervioso o de mal humor si los progenitores les impiden navegar.

Cabe señalar, que los juegos digitales se abren camino entreverados con objetivos empresariales bajo el argumento de que incentivan el aprendizaje de los menores (Spitzer, 2013). No obstante, son muchas las voces de expertos que alertan de las repercusiones que puede tener para los niños y los jóvenes un mal uso de la tecnología.

A tenor de lo anterior, el ámbito educativo debe examinar y valorar tanto los beneficios como los riesgos. La Encuesta sobre Equipamiento y Uso de la TICS en los hogares elaborada por el Instituto Nacional de Estadística en 2019concluía que, entre los hogares españoles con hijos, había 792.048 sin dispositivos; y más recientemente en el año 2020 el uso de las TIC en menores sigue estando cada vez más extendida. Los datos indican que la conexión a internet es prácticamente universal. De hecho, el $81,4 \%$ de los hogares con al menos un miembro de 16 a 74 años dispone de algún tipo de ordenador (de sobremesa, portátil, tablet...), lo que supone un aumento de 0,5 puntos respecto a 2019 . Respecto al tipo de dispositivo utilizado, el 76,2\% cuenta con ordenadores de sobremesa o portátiles, y el $58,4 \%$ con tablets. El teléfono móvil está presente en casi la totalidad de los hogares (el $99,5 \%$, con una subida de un punto respecto a 2019). Por su parte, el uso diario de las TIC en jóvenes de 16 a 24 años es del $82,4 \%$ en hombres y el $83,8 \%$ en mujeres (INE, 2020). Por otro lado, respecto a las habilidades digitales, la encuesta sitúa al grupo de edad de 16 a 24 años con mayores habilidades (el $75,7 \%$ las tiene avanzadas).

En la investigación llevada a cabo por Rial et al. (2015) se pone de manifiesto que aproximadamente uno de cada cuatro escolares de 11 a 17 años podría estar haciendo un "uso problemático" de la red. Tanto su investigación como la realizada anteriormente por García, et al. (2014) han examinado el tiempo de uso y el efecto negativo que ocasiona en el rendimiento académico. Ambos estudios recalcan que un $12.5 \%$ de adolescentes afirman haber disminuido su rendimiento escolar y un $4.5 \%$ reconoce haber reducido el tiempo que pasa con sus amigos a causa de las TIC.

En términos valorativos, Sharif y Sargent (2006) destacan en sus conclusiones también que el uso continuo de videojuegos repercute negativamente en el rendimiento escolar.

Por otra parte, un aspecto digno de destacar es la diferencia en el uso de las TIC que pueda existir entre chicos y chicas. En esa línea de resultados se encuentra el estudio llevado a cabo por Berríos y Buxarrais (2005) que consideran que el atractivo principal de los chicos es el ocio lúdico, principalmente a través de los videojuegos, mientras que los intereses de las chicas giran en torno a la comunicación de índole social por medio de conversaciones con el móvil. A estas mismas conclusiones llegaron, varios años más tarde los trabajos de Villadangos y Labrador (2009) y García et al. (2014).

Por otro lado, hay corrientes que encuentran similitudes en ciertas conductas con aquellas que son consideradas como adicción a sustancias. Estas son: dependencia psicológica, tolerancia (aumento del tiempo para obtener similar satisfacción), síndrome de abstinencia (malestar psicológico). Además, algunos estudios advierten que ciertas vulnerabilidades genéticas son compartidas tanto en conductas adictivas como en aquellas con adicciones a sustancias, así como en determinadas características cerebrales y ciertas manifestaciones psicopatológicas (Ginige, 2017). A este respecto, Young ya hizo referencia en 1999 a un cierto deterioro en el control de su uso. Defendía de forma taxativa que la adicción a internet estaba acompañada de síntomas cognitivos, conductuales y fisiológicos.

Por todo ello podemos sintetizar lo expuesto hasta el momento afirmando que existen discrepancias notables respecto a la relación existente entre el rendimiento escolar y el mal uso de las TIC. Ciertos autores objetan el resultado de estos planteamientos críticos e incluso afirman que pueden erigirse en un motor fundamental para aportar más eficacia al aprendizaje y facilitar el rendimiento escolar. Paralelamente existen otros estudios centrados en el uso y abuso de las TIC que advierten por el contrario cómo pueden llegar a producir adicción en los jóvenes, destacando los factores más relevantes que entran en juego. Sin embargo, no existe un consenso claro a la hora de determinar los límites entre uso, abuso y adicción. En cualquier caso, tanto unos como otros son conscientes de que la irrupción de las nuevas tecnologías que ha tenido lugar en las aulas en los últimos años, no ha tenido precedentes históricos y que va a ser relevante.

\section{CONTEXTO}

\subsection{Necesidad de su realización}

De acuerdo con la tesis defendida por Pedrero-Pérez et al. (2012) no existe acuerdo ni evidencia suficiente en la comunidad científica, como para sustentar de forma taxativa la adicción a internet. No obstante, sí puede afirmarse con certeza de que su uso puede ser excesivo, y que por ese motivo puede ocasionar un descontrol en la conducta en ámbitos diversos de la vida (Elhai et al., 2017). Los adolescentes constituyen un sector poblacional vulnerable, que ha recibido en los últimos años un gran impacto de la tecnología. De ahí la conveniencia de profundizar acerca del uso de las TIC entre jóvenes adolescentes. Consideramos que a pesar de que existen trabajos parciales al respecto, resulta conveniente llevar a cabo una revisión y actualización de esos trabajos debido a la rápida evolución tecnológica que experimentamos día a día.

De hecho, tal y como apuntábamos surgen cuestiones de gran calado en el ámbito educativo que invitan a la reflexión. Por una parte, existen defensores de las nuevas tecnologías que argumentan a favor de un impacto positivo y significativo de las variables TIC, y por otra parte encontramos un grupo de investigadores que no advierte efecto alguno de las variables TIC en el rendimiento escolar. A su vez, aunque en menor medida, algunos investigadores interpretan que existe una incidencia negativa de las TIC sobre los logros académicos. ¿Cómo es posible esa divergencia?

\subsection{Objetivos}

En este contexto, el objetivo de este trabajo es medir el uso y frecuencia de las nuevas Tecnologías de la Información y la Comunicación como hábito diario de ocio en una muestra de estudiantes españoles de bachillerato, explorando las posibles diferencias de género, cualitativas y cuantitativas, así como valorando su posible asociación con una disminución del rendimiento escolar. 
Como objetivo principal estudiaremos si la adicción a internet afecta al rendimiento académico de los adolescentes en función del sexo. La hipótesis que se pretende contrastar es: si el grado de adicción a internet afecta negativamente al rendimiento académico en función del sexo de los adolescentes.

\section{DESCRIPCIÓN}

\subsection{Participantes}

La muestra total estuvo compuesta por un total de 72 estudiantes pertenecientes a un centro educativo de enseñanza concertada en el curso escolar 2019-20. Se tomó una muestra no probabilística con edades comprendidas entre 16 y 18 años $(\mathrm{M}=16,1 ; \mathrm{DT}=, 491)$ de primero y segundo de bachillerato. (Tabla 1).

Tabla 1

Distribución de los participantes en función del sexo y del nivel de estudios

\begin{tabular}{lllc}
\hline & Chicas & Chicos & $\begin{array}{c}\text { Totales } \\
\text { \% }\end{array}$ \\
\hline & 14 & 14 & 28 \\
$\begin{array}{c}\mathbf{1}^{\text {o }} \\
\text { Bachillerato }\end{array}$ & & $38,88 \%$ \\
$\begin{array}{c}\mathbf{2}^{\mathbf{0}} \\
\text { Bachillerato }\end{array}$ & 30 & 14 & 44 \\
Porcentajes & $61,11 \%$ & $38,88 \%$ & $61,11 \%$ \\
& & & 72 \\
\hline
\end{tabular}

\subsection{Instrumentos}

Los instrumentos y medidas utilizados se encuentran en la Tabla 2.

\section{Tabla 2}

Instrumentos utilizados

\section{Instrumentos}

Cuestionario de Detección de Nuevas adicciones (DENA) de (Labrador et al., 2013)

El Test de Adicción a Internet (IAT) de Young (1999)
Análisis de la frecuencia del uso de las TIC: Internet, Móvil y videojuegos, en función del género.

\section{Evaluación}

del comportamiento adictivo en 4 niveles: sin adicción, leve, moderado y severo.

\section{Características}

\subsubsection{Cuestionario de Detección de Nuevas adicciones (DENA, 2013).}

Para analizar la frecuencia de uso de internet, teléfono móvil y videojuegos, se utilizó un instrumento de evaluación que mide tanto el uso de las TIC como la percepción subjetiva de posibles problemas derivados de su utilización.
Respecto a las características del cuestionario, los datos aportados por el trabajo de Labrador et al. (2013) indican respecto a la fiabilidad, en general, un alfa de Crombach adecuado en especial la escala total (.876).

\subsubsection{El Test de Adicción a Internet (IAT) de Young (1999):}

Este instrumento es el primero que fue validado con el fin de evaluar la adicción a internet (Widyanto y McMurren, 2004). Diversos estudios han mostrado que el IAT es una medida confiable que cubre las características claves del uso patológico online.

El IAT puede ser utilizado en contextos ambulatorios o residenciales, y también puede ser adaptado para satisfacer las necesidades del encuadre clínico.

Se le reconoce una alta fiabilidad y una fuerte consistencia interna. Tiene una Alfa de Cronbach (1951) que oscila entre el 0’89 y 0'91 y en su versión online de entre 0'83 y 0'91.

\subsection{Procedimiento}

La primera toma de contacto tuvo lugar a través de una reunión con el director del centro educativo, que dio su aprobación, y posteriormente, se coordinaron las agendas. Después se concertó un día y hora determinados para explicar a los participantes la naturaleza y finalidad del estudio, y una vez recogidos todos los cuestionarios cumplimentados y los consentimientos informados se asignó un código numérico y se introdujeron los datos de cada ejemplar, en el programa estadístico SPPS, versión 19.0.

\section{Resultados}

Se utilizó en primer lugar un diseño de encuestas transversal para comprobar a nivel descriptivo el uso que hacen los adolescentes de las TIC y si existen diferencias de sexo en su uso.

En segundo lugar, con el fin de contrastar nuestra hipótesis se utilizó un diseño ex post facto prospectivo complejo con dos VVII: adicción a internet $\mathrm{y}$ sexo; $\mathrm{y}$, como variable dependiente: rendimiento académico.

La figura 1 muestra el tiempo de conexión a internet a lo largo del día de los participantes.

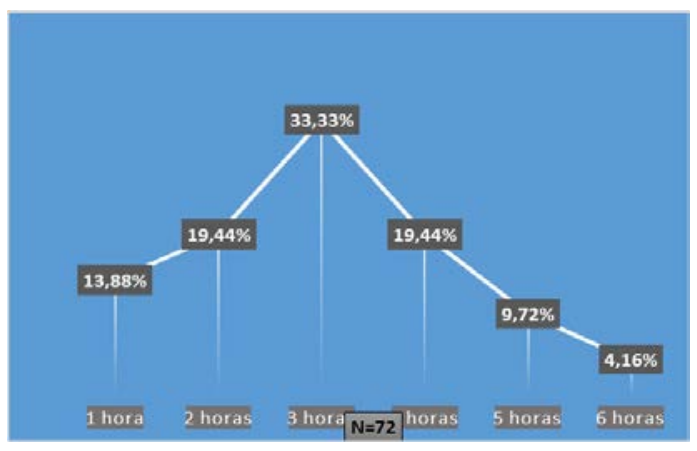

Figura 1: Tiempo de conexión a internet a lo largo del día de los participantes.

Cuando se les pregunta directamente si alguna vez has intentado desconectarte a internet y no lo has conseguido, los 
resultados muestran que el $46,42 \%$ de los chicos y el $75 \%$ chicas sí han tenido dificultades. $(\chi 2(2,72)=6.672, p=.036)$, el estadístico "chi cuadrado" indica que hay diferencias significativas. (Figura 2)

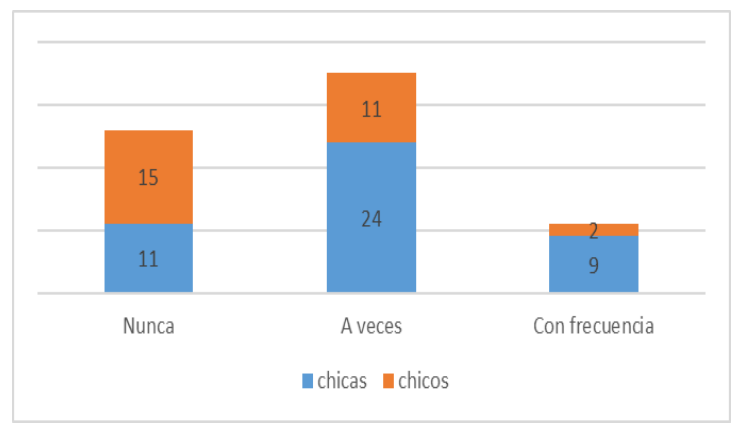

Figura 2: Dificultades para desconectarse a internet en función del género.

Respecto al uso de la telefonía móvil se detectaron diferencias de género, la figura 3 muestra el uso.

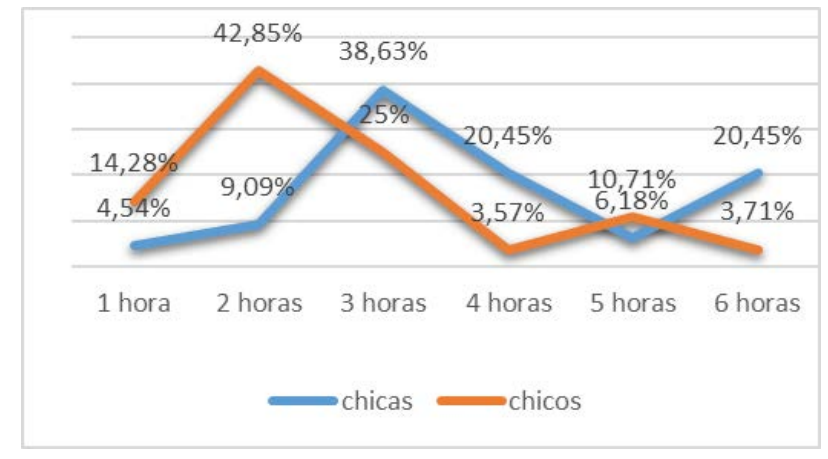

Figura 3: Uso del teléfono móvil en función del género.

La utilización del ocio digital a través de videojuegos se muestra en la Figura 4.

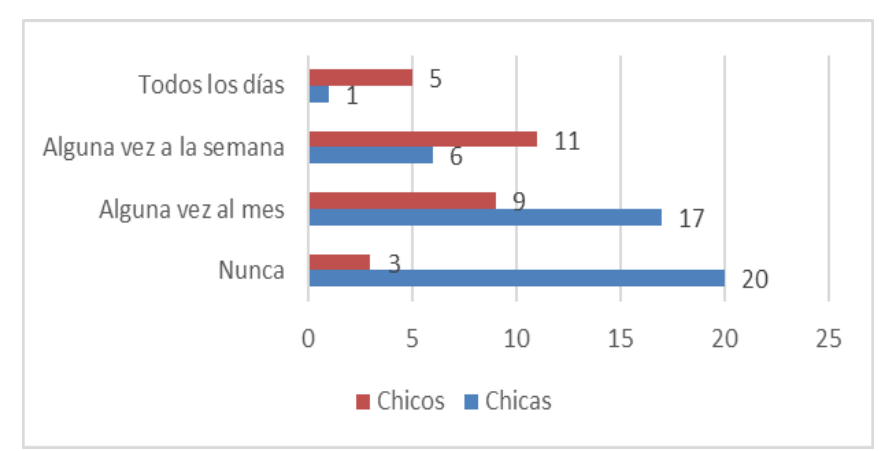

Figura 4: Uso de los videojuegos en función del género.

Respecto al lugar donde hacen uso de las TIC:

a) El $86,1 \%$ se conecta a internet en casa y el $9,70 \%$ acude a espacios públicos para realizar la conexión (biblioteca, cibercafés). b) El 70,80\% decide jugar a videojuegos en casa frente al 15,30\% que lo hace fuera del hogar.

La Tabla 3 recoge los resultados del análisis del cuestionario de adicción (IAT). Young (1999)

Tabla 3

Niveles de adicción a internet en relación al sexo

\begin{tabular}{cccc}
\hline Niveles IAT & Frecuencia & $\%$ & $\begin{array}{c}\% \\
\text { acumulado }\end{array}$ \\
\hline Rango Normal & & $58,3 \%$ & \\
sin adicción & 43 & $58,13 \%$ & $58,3 \%$ \\
Chicas & 25 & $41,86 \%$ & \\
Chicos & 18 & & \\
\hline Adicción leve & 23 & $33,3 \%$ & \\
Chicas & 15 & $65,21 \%$ & $91,7 \%$ \\
Chicos & 8 & $34,78 \%$ & \\
\hline Adicción & 6 & & \\
moderada & 4 & $8,3 \%$ & $100 \%$ \\
Chicas & 2 & $66,66 \%$ & \\
Chicos & & $33,33 \%$ & $100 \%$ \\
\hline Total & 72 & $100 \%$ & \\
\hline
\end{tabular}

De acuerdo con los datos obtenidos, hemos considerado conveniente agrupar los resultados de este cuestionario (IAT) en dos grupos: el grupo 1, con los participantes sin adicción $(\mathrm{N}=43)$ y el grupo 2 con aquellos que han obtenido niveles de adicción leve y moderada $(\mathrm{N}=29)$

La Tabla 4 muestra los resultados obtenidos del Anova Bifactorial. No existe interacción entre la adicción y el género, es decir, el género no influye en el efecto de la adicción en el rendimiento.

\section{Tabla 4}

Resultados del Anova Bifactorial

\begin{tabular}{|c|c|c|c|c|c|}
\hline Origen & $\begin{array}{l}\text { Tipo III } \\
\text { de suma } \\
\text { de } \\
\text { cuadrad } \\
\text { os }\end{array}$ & gl & $\begin{array}{c}\text { Media } \\
\text { cuadráti } \\
\text { ca }\end{array}$ & $\mathrm{F}$ & Sig. \\
\hline $\begin{array}{l}\text { Modelo } \\
\text { corregido }\end{array}$ & $4,628^{a}$ & 3 & 1,543 & ,698 &, 557 \\
\hline Adicción & 3,801 & 1 & 3,801 & 1,719 &, 194 \\
\hline GÉNERO &, 005 & 1 &, 005 & ,002 & ,960 \\
\hline $\begin{array}{c}\text { Adicción } \\
*\end{array}$ & 1,519 & 1 & 1,519 & ,687 &, 410 \\
\hline $\begin{array}{c}\text { GÉNERO } \\
\text { Error } \\
\end{array}$ & 150,351 & 68 & 2,211 & & \\
\hline $\begin{array}{r}\text { Var } \\
\mathrm{R} \text { al cu } \\
\end{array}$ & $\begin{array}{l}\text { Iable depen } \\
\text { adrado }=, 0\end{array}$ & $\begin{array}{l}\text { ente: } \\
(\mathrm{R} \mathrm{a}\end{array}$ & $\begin{array}{l}\text { RENDIM } \\
\text { l cuadrado }\end{array}$ & $\begin{array}{l}\text { ENTO } \\
\text { ustada = }\end{array}$ & $-, 013)$ \\
\hline
\end{tabular}

20-22 Octubre 2021, Madrid, ESPAÑA

VI Congreso Internacional sobre Aprendizaje, Innovación y Cooperación (CINAIC 2021) 
La tabla 5 y figura 5 recogen las medias entre rendimiento y adicción.

\section{Tabla 5}

\begin{tabular}{cr|r|r|r} 
Adicción & N & Media & \multicolumn{1}{c}{$\begin{array}{c}\text { Desv. } \\
\text { Desviación }\end{array}$} & \multicolumn{1}{c}{$\begin{array}{c}\text { Desv. Error } \\
\text { promedio }\end{array}$} \\
\hline 1 & 43 & 6,4674 & 1,65444 &, 25230 \\
\hline 2 & 29 & 6,0531 & 1,15022 &, 21359 \\
\hline
\end{tabular}

Medias del rendimiento en el grupo 1 (sin adición) y el grupo 2 (con adicción)

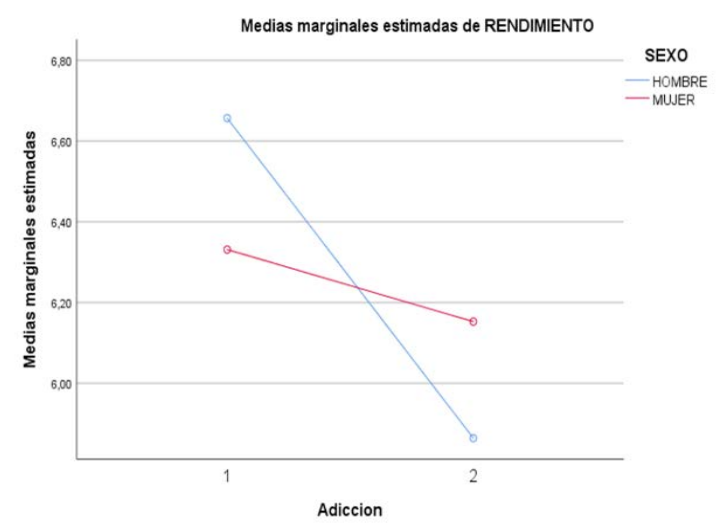

Figura 5: Medias entre adicción, rendimiento y género.

Finalmente, la Tabla 6 muestra los resultados obtenidos en la T de Student, los cuales no detectan diferencias significativas en las medias del rendimiento de los dos grupos. Este resultado está en consonancia con los resultados anteriores en la Tabla 5.

\section{Tabla 6}

Resultados entre los niveles de adicción a internet y el rendimiento

\begin{tabular}{|c|c|c|c|c|c|c|}
\hline & \multicolumn{4}{|c|}{$\begin{array}{r}\text { Prueba de Levene de igualdad } \\
\text { de varianza }\end{array}$} & \multirow[b]{2}{*}{$\mathrm{gl}$} & \multirow[b]{2}{*}{$\begin{array}{r}\text { sig. } \\
\text { (bilateral), }\end{array}$} \\
\hline & & $\mathrm{F}$ & sig & $\mathrm{t}$ & & \\
\hline Rendimiento & $\begin{array}{r}\text { Se } \\
\text { asumen } \\
\text { varianzas } \\
\text { iguales }\end{array}$ & $\begin{array}{r}3,67 \\
9\end{array}$ &, 059 & 1,170 & 70 & 246 \\
\hline
\end{tabular}

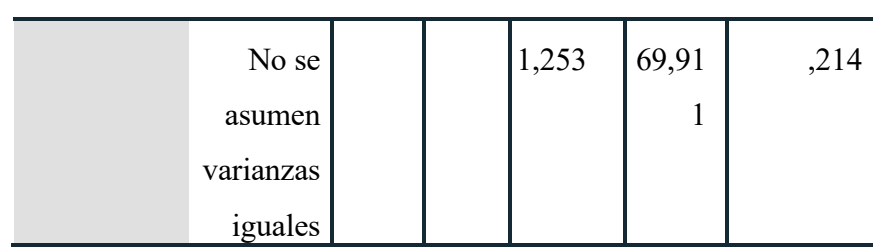

\section{CONCLUSIONES}

En concordancia con la literatura revisada la hipótesis no se confirma, ya que los resultados obtenidos constatan que la utilización o el abuso de las TIC no están relacionados con el rendimiento escolar. Concretamente, hemos hallado que el empleo de las TIC no afecta al rendimiento escolar en los adolescentes y que existen diferencias de sexo manifiestas en el uso de videojuegos y del móvil. Tanto las chicas como los chicos se conectan a internet todos los días, siendo las chicas más proclives a tener problemas para desconectarse.

Los resultados obtenidos indican que la mayor parte de la muestra se conecta a las TIC en casa. La mitad de la muestra se encuentra en niveles de normalidad, sin indicios de adicción. Los datos obtenidos no manifiestan niveles de adicción severa, sin embargo, sí se han encontrado niveles de adicción leves, siendo estos algo más acentuados en el grupo de las chicas. La relación entre la variable rendimiento y la variable utilización y abuso se encuentra principalmente entre los niveles leves o moderados.

No obstante, el análisis llevado a cabo en este estudio corrobora algunos trabajos previos consultados, que señalan que aún no existe consenso ni evidencia suficiente como para sustentar adicción a las TIC (Pedrero-Pérez et al., 2012). Los resultados encontrados permiten confirmar que la conexión permanente con sus iguales sirve a la generación interactiva en su trabajo escolar, y no hay evidencias de déficit en rendimiento tal y como constataron, Bringué y Sádaba (2009). No obstante, quizás podrían producirse consecuencias negativas si las redes sociales fueran mal empleadas, como precisa Mejía Zambrano (2015).

En cuanto a futuros trabajos de investigación, conviene precisar que la población que ha sido objeto de estudio se sitúa en una etapa vulnerable y muy importante para su desarrollo. Por tanto, convendría disponer del índice socioemocional de los participantes, así como del nivel en conocimientos digitales de los propios participantes y de su entorno familiar y social directo. Así mismo, podría resultar beneficioso concretar líneas terapéuticas o posibles tratamientos que pudieran paliar conductas adictivas.

Los resultados de nuestro estudio sugieren la necesidad de establecer programas de prevención dirigidos a los padres o tutores legales o al profesorado en general, para el uso seguro y responsable de Internet. Del mismo modo, dejan constancia de que, si los profesionales de la psicología conocieran el perfil psicopatológico de niños y adolescentes con uso problemático a las TIC, eso permitiría realizar intervenciones diferenciales y más específicas. 
A modo de conclusión y para finalizar podemos afirmar que los resultados no mostraron diferencias significativas entre las variables, rendimiento y adicción. Tampoco entre adicción y sexo y rendimiento. Cabe señalar adicionalmente que la variable de género es independiente del rendimiento. En definitiva, la relación entre adicción y rendimiento fue débil y negativa. Los resultados obtenidos en este trabajo son similares a los hallados en estudios realizados previamente.En definitiva, los resultados obtenidos en nuestra investigación nos inducen a plantear el desarrollo de programas de prevención dirigidos a los padres o tutores legales o al profesorado en general, para el uso seguro y responsable de Internet.

\section{REFERENCIAS}

Ballesteros, J. C., y Megías, I. (2015). Jóvenes en la red: un selfi. Centro Reina Sofía sobre adolescencia y juventud. Fundación de Ayuda contra la Drogadicción (FAD). https://doi.org/10.5281/zenodo.3653963

Berríos, LL., y Buxarrais, M.R. (2005). Las tecnologías de la información y la comunicación (TIC) y los adolescentes. Algunos datos. Monografías virtuales- Ciudadanía, democracia y valores en sociedades plurales- Valores y tecnologías de la información y comunicación. OEI.https://www.oei.es/historico/valores $2 /$ monografias/m onografia05/reflexion05.htm

Bringué, X., y Sádaba, C. (2009). La Generación interactiva en España. Niños y adolescentes ante las pantallas. ESE: Estudios sobre educación, 18, 319-320.

Castañeda, L., y Adell, J. (2013). Entornos Personales de Aprendizaje: claves para el ecosistema educativo en red. Alcoy: Marfil.

Elhai, J. D., Dvorak, R. D., Levine, J. C.. y Hall, B. J. (2017). Problematic smartphone use: a conceptual overview and systematic review of relations with anxiety and depression psychopathology. Journal of Affective Disorders, 207, 251-259.

García, B., De Ayala López, M. y García A. (2014). Los riesgos de los adolescentes en Internet: los menores como actores y víctimas de los peligros de Internet. Revista latina de comunicación social,69(4), 462-485. https://doi.org//10.4185/RLCS-2014-1020

Ginige, P. (2017, pp. 141-163). Child and adolescent mental health. IntechOpen.

Instituto Nacional de Estadística (INE) (2020). Encuesta sobre Equipamiento y Uso de Tecnologías de Información y Comunicación en los Hogares.

Labrador, F.J., Villadangos, S.M., Crespo, M., y Becoña, E. (2013). Desarrollo y validación del cuestionario de uso problemático de nuevas tecnologías (UPNT). Anales de psicología (Servicio de publicaciones de la Universidad de Murcia), $29 \quad$ (3), $\quad 836-847$. https://doi.org/10.6018/analesps.29.3.159291

Martínez, N. y González, E. (2018). Adolescentes y redes sociales: panorámica general sobre el uso, el tiempo y los riesgos. Journal for Educators, Teachers and Trainers, $9(1), 42-54$.
Mejía Zambrano, V. J. (2015). Análisis de la influencia de las Redes Sociales en la formación de los jóvenes de los colegios del Cantón Yaguachi. [Tesis doctoral, Universidad de Guayaquil. (Ecuador)]. Repositorio Universidad de Guayaquil.

Pedrero-Pérez, E. J., Rodríguez-Monje, M. T. y Ruiz-Sánchez de León, J. M. (2012). Adicción o abuso del teléfono móvil. Revisión de la literatura. Adicciones, 24, 139152. http://www.adicciones.es/index.php/adicciones/article/v iew/107

Prensky, M. (2010). Nativos e inmigrantes digitales. Institución Educativa Sek

Rial, A., Golpe, S., Gómez, P., y Barreiro, C. (2015). Variables asociadas al uso problemático de Internet entre adolescentes. Health and Addictions, 15(1), 25-38.

Robles, J., y Torres-Albero, C. (2016). Brecha y desigualdad digital, en C. Torres-Albero (2015). Situación Social, 107-130. Centro de Investigaciones Sociológicas.

Sharif, I., y Sargent, J. D. (2006). Association between television, movie, and video game exposure and school performance. Pediatrics, 118(4), 1061-1070.

Spitzer,M. (2013). Demencia Digital. Ediciones B.

Torres-Albero, C., Robles, J., y De Marzo, S. (2017). Revisión analítica del modelo de aceptación de la tecnología. El cambio tecnológico. Papers, Revista de Sociología, 102 (1) ,5-27

Tourón, J., y Santiago, R. (2013). Atención a la diversidad y desarrollo del talento en el aula. El modelo DT-PI y las tecnologías en la implantación de la flexibilidad curricular y el aprendizaje al propio ritmo. Revista española de pedagogía, 71(256),441-459.

Villadangos, S.M., y Labrador, F.J. (2009). Menores y nuevas tecnologías (NT): ¿Uso o abuso? Anuario de Psicología Clínica $y$ de la Salud,5, 75-83. https://institucional.us.es/apcs/doc/APCS_5_esp_7583.pdf

Widyanto, L., y McMurran, M. (2004). The psychometric properties of the internet addiction test. Cyberpsychology y behavior, 7(4), 443-450.

Young, K. S. (1999). Internet addiction: symptoms, evaluation and treatment. Innovations in clinical practice: A source book, 17(17), 351-35 